\title{
FILLING GAPS IN CULTURAL HERITAGE DOCUMENTATION BY 3D PHOTOGRAPHY
}

\author{
W. Schuhr ${ }^{\mathrm{a}, *}$ and J.D. Lee ${ }^{\mathrm{b}}$ \\ ${ }^{a}$ University of Applied Sciences Magdeburg, Germany - (Schuhr3D@ hotmail.com) \\ ${ }^{\mathrm{b}}$ Kumoh National University of Technology (kit), Gumi, Republic of Korea
}

25th International CIPA Symposium, session E-4, Taipei, 2015

KEY WORDS: tangible \& intangible C.H., Archives of 3D photography, UAV-platforms, Monument Information System (MIS), 3D-Technology, Internet/Web, lasered crystals, 3D printer

\begin{abstract}
:
This contribution promotes 3D photography as an important tool to obtain objective object information. Keeping mainly in mind World Heritage documentation as well as Heritage protection, it is another intention of this paper, to stimulate the interest in applications of 3D photography for professionals as well as for amateurs.

In addition this is also an activity report of the international CIPA task group 3.

The main part of this paper starts with "Digging the treasure of existing international 3D photography". This does not only belong to tangible but also to intangible Cultural Heritage. 3D photography clearly supports the recording, the visualization, the preservation and the restoration of architectural and archaeological objects. Therefore the use of 3D photography in C.H. should increase on an international level. The presented samples in 3D represent a voluminous, almost partly "forgotten treasure" of international archives for 3D photography.

The next chapter is on "Promoting new 3D photography in Cultural Heritage". Though 3D photographs are a well-established basic photographic and photogrammetric tool, even suited to provide "near real" documentation, they are still a matter of research and improvement. Beside the use of 3D cameras even single lenses cameras are very much suited for photographic 3D documentation purposes in Cultural Heritage.

Currently at the Faculty of Civil Engineering of the University of Applied Sciences Magdeburg-Stendal, low altitude aerial photography is exposed from a maximum height of $13 \mathrm{~m}$, using a hand hold carbon telescope rod. The use of this "huge selfie stick" is also an (international) recommendation, to expose high resolution 3D photography of monuments under expedition conditions. In addition to the carbon rod recently a captive balloon and a hexacopter UAV-platform is in use, mainly to take better synoptically (extremely low altitude, ground truth) aerial photography.

Additional experiments with respect to "easy geometry" and to multistage concepts of 3D photographs in Cultural Heritage just started. Furthermore a revised list of the 3D visualization principles, claiming completeness, has been carried out.

Beside others in an outlook

*It is highly recommended, to list every historical and current stereo view with relevance to Cultural Heritage in a global Monument Information System (MIS), like in google earth.

*3D photographs seem to be very suited, to complete and/or at least partly to replace manual archaeological sketches. In this concern the still underestimated 3D effect will be demonstrated, which even allows, e.g., the spatial perception of extremely small scratches etc...

*A consequent dealing with 3D Technology even seems to indicate, currently we experience the beginning of a new age of "real 3DPC-screens", which at least could add or even partly replace the conventional 2D screens.

Here the spatial visualization is verified without glasses in an all-around vitreous body.

In this respect nowadays widespread lasered crystals showing monuments are identified as "Early Bird" 3D products, which, due to low resolution and contrast and due to lack of color, currently might even remember to the status of the invention of photography by Niepce (1827), but seem to promise a great future also in 3D Cultural Heritage documentation.

*Last not least 3D printers more and more seem to conquer the IT-market, obviously showing an international competition.
\end{abstract}

\section{INTRODUCTION}

According to the 25th CIPA Symposium motto "Filling the Gaps - Towards a Globalization Era," this contribution is concentrating on promoting 3D photography as an important tool to obtain objective object information in the field of Cultural Heritage.

Keeping mainly in mind World Heritage protection as well as Heritage documentation, it is another intention of this paper, to stimulate the interest in applications of 3D photography not only for professionals but also for amateurs.

Furthermore this is also an activity report of the international CIPA task group 3.

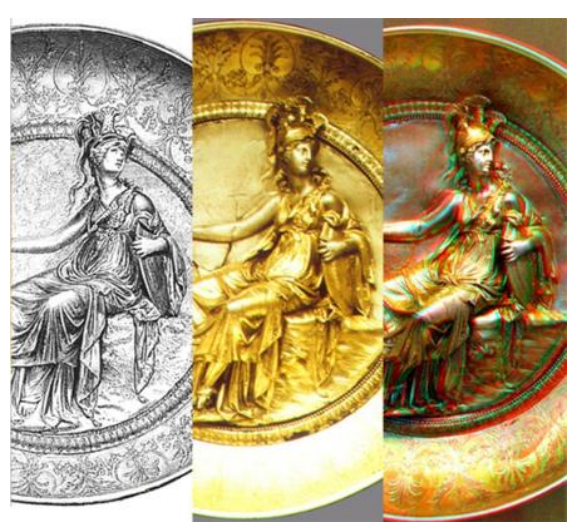

Figure 1. Sample showing the advantage of 3D photographs in comparison to a drawing and to a 2D photograph (object: Athena show-piece of the famous Hildesheim Treasure) 


\section{DIGGING THE TREASURE OF EXISTING INTERNATIONAL 3D PHOTOGRAPHY}

Within this frame presented (mainly historic) masterpieces of 3D imagery, not only show tangible (see fig. 1 to 3,9 and 12 to14) but also intangible (see fig. 2 to 3 ) Cultural Heritage.

Obviously this type of 3D photography supports the recording, the visualization, the preservation and the restoration of architectural and archaeological objects, including excavation documentation, virtual museum requirements, spatial structure enhancement in rock arts, aiding interpretation and/or serving as educational tool, etc.

Therefore it is highly recommended, practical applications of 3D photography in cultural heritage should increase on an international level.

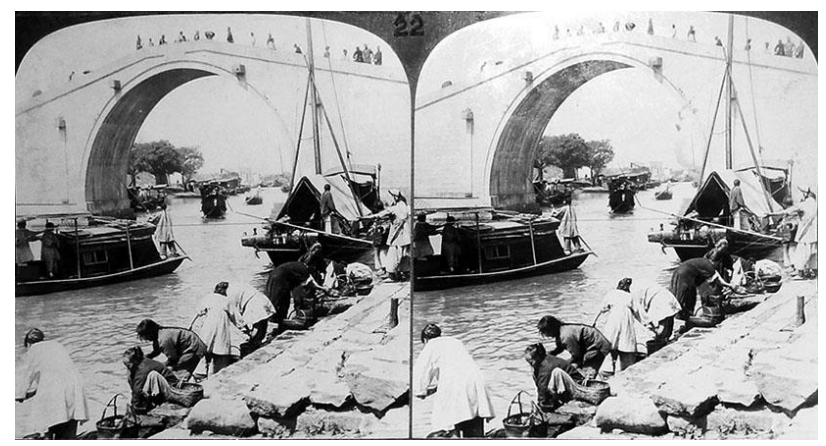

Figure 2. Historic masterpieces of scenery at women bride of the Imperial Canal near Soo-Chow (China) (by Underwood \&Underwood, approx. 1900)

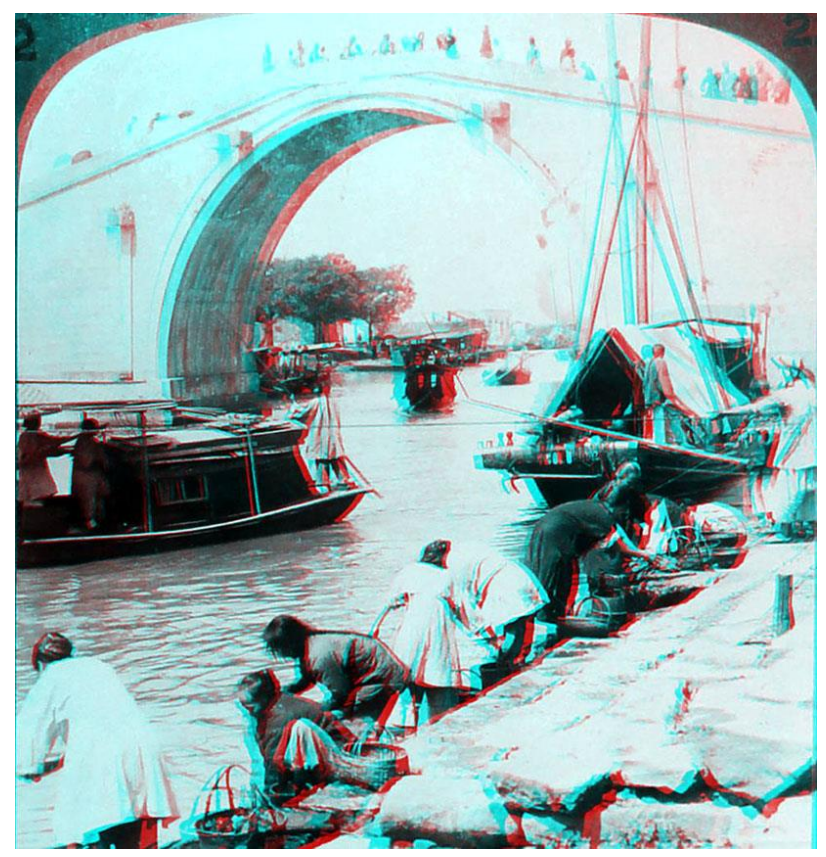

Figure 3. Anaglyphs presentation of figure 2, showing partly tangible but also intangible cultural aspects

The samples of 3D photographs (in figure $2 \& 3$ ) represent a voluminous, almost partly "forgotten treasure" of international archives for 3D photography, like, e.g., the collection of the California Museum for Photography (CMP) in particular, which possesses more than 1 million analog stereo negatives and stereo prints, see figure 4 . The digitization of the analog 3D photographs of the Keystone-Mast-Collection is in progress.

The Korean HAN collection is another sample of an archive for 3D photographs (see figure 5).

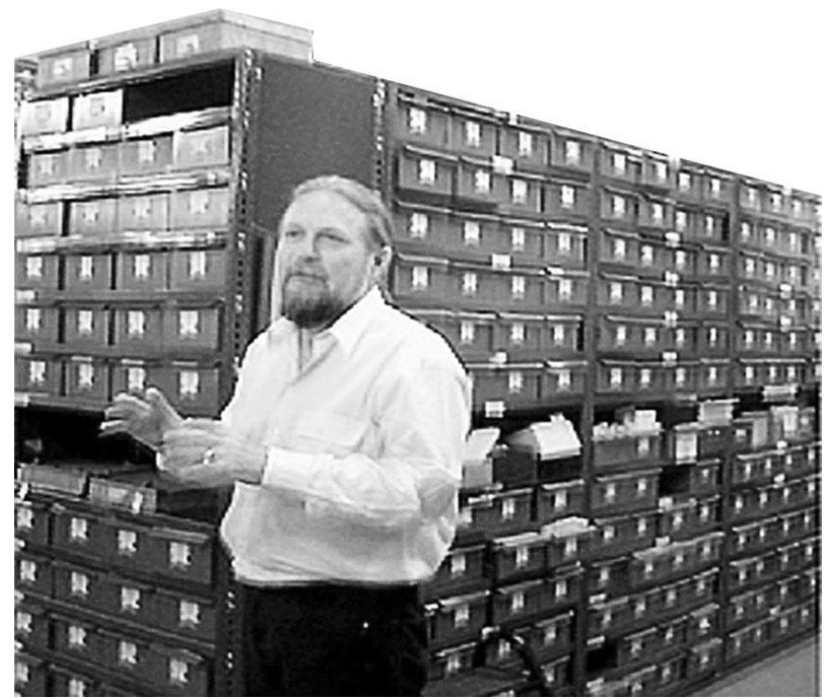

Figure 4. Inside the archive of the UCR/CMP Keystone-MastCollection at the CMP (photograph by Francois LeBlanc (GCI))

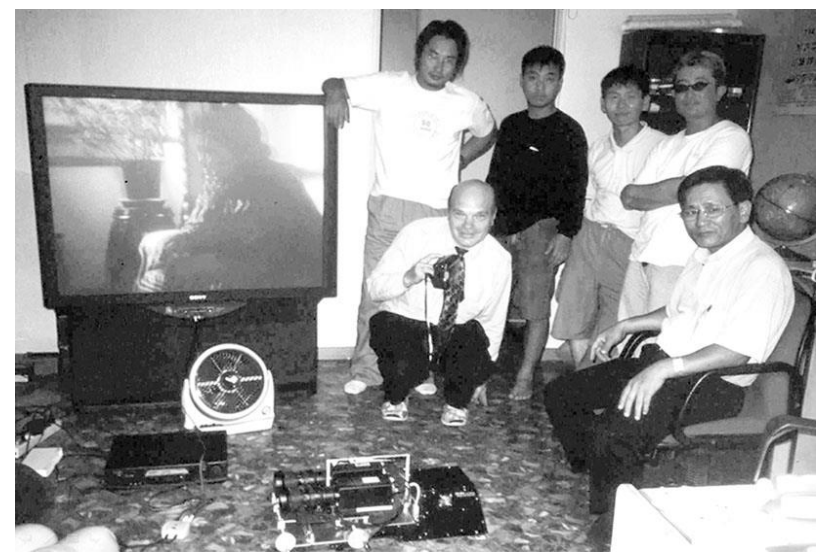

Figure 5. Early visit of the 1st author at the HAN Archive of 3D photographs (including early $3 \mathrm{D}$ videos)

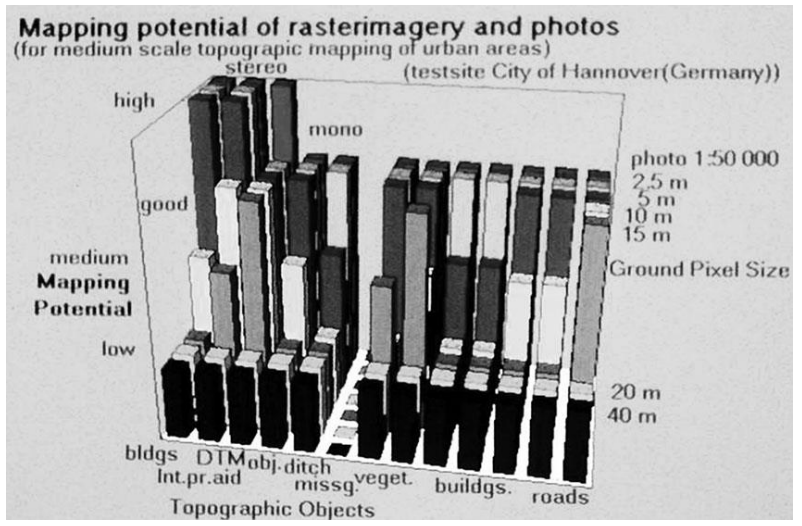

Figure 6. Sample for a 1st research result in 3D photography: The minimum digitization rate for analog $9 \times 9$ inch stereo views $=500$ Dots $/$ inch $(=2.5 \mathrm{~m} / 50000=100 \mathrm{inch} / 50000=1 \mathrm{inch} / 500)$ 
Referring again to the CIPA Symposium motto "Filling the Gaps - Towards a Globalization Era," one important goal in the field of 3D photography is, to search for a complete international list of existing archives for 3D photography, including access conditions. This also will be backed up by a proposed cooperation with the International Stereoscopic Union (ISU).

In this respect the authors recently even follow a trace to discover another extremely rare photo plasticon device of the 1920th. Beside others, within this part also the question after the optimum digitization rate for archived analog historic 3D imagery will be analyzed. A first research result for an optimum digitization rate for analog 9x9 inch stereo views resulted into 500 dots per inch, see figure 6.

\section{PROMOTING NEW 3D PHOTOGRAPHY IN CULTURAL HERITAGE}

Though 3D photographs are a well-established basic photographic and photogrammetric tool, even suited to provide "near real" documentation, they are still a matter of research and improvement.

To improve praxis in 3D photography own experiences according to "Learning by Doing" is essential.

For first steps the use of 3D cameras showing a fixed $6 \mathrm{~cm}$ base, like, e.g., the Fuji Finepix Real 3D camera, which also allows to take 3D movie sequences, not only for intangible cultural heritage, is recommended within a limited range.

For photographic 3D documentation purposes in Cultural Heritage even single lenses cameras are very suited. It is highly recommended, not only to expose just 2 stereo photographs but to take a queue of, e.g., 7 and more photographs showing different base lengths.

This allows to obtain 3D photographs, considering different aspects, applying, e.g., the Stereophotomaker software, as the standards for exposing and processing 3D Cultural Heritage photographs are still a matter of improvement.

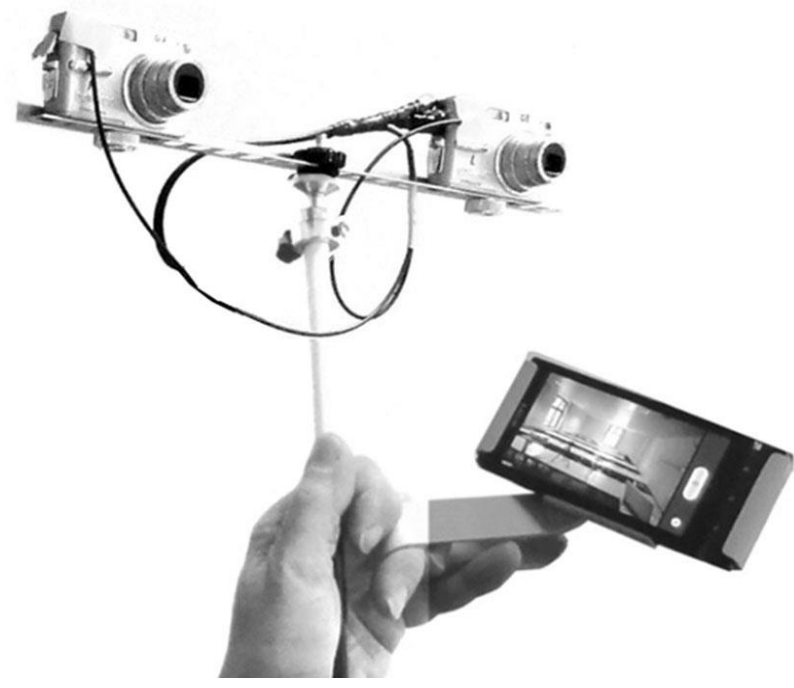

Figure 7. X-Lite carbon telescope staff carrying WLAN capable stereo-cameras with smartphone App based remote control (technical study, partly carried out by St. Kiel of the University of Appl. Sc. Magdeburg)
Currently at the Faculty of Civil Engineering of the University of Applied sciences Magdeburg-Stendal, low altitude aerial photography is exposed (via a WLAN capable camera with smartphone App) from a maximum height of $13 \mathrm{~m}$, using a hand hold carbon telescope rod. The use of this "huge selfie stick" is also a recommendation, to expose high resolution 3D photography of monuments under expedition conditions, see figure 7. Down in Magdeburg in addition to the carbon rod a captive balloon and nowadays of course, also a hexacopter UAV- platform is in use, mainly to take better (extremely low altitude, ground truth) synoptically aerial 3D photography, see figure 8 .

Additional experiments with respect to multistage concepts of 3D photographs in Cultural heritage, like combining 3D imagery showing differences in focus, daytime, spectral characteristic etc., just started.

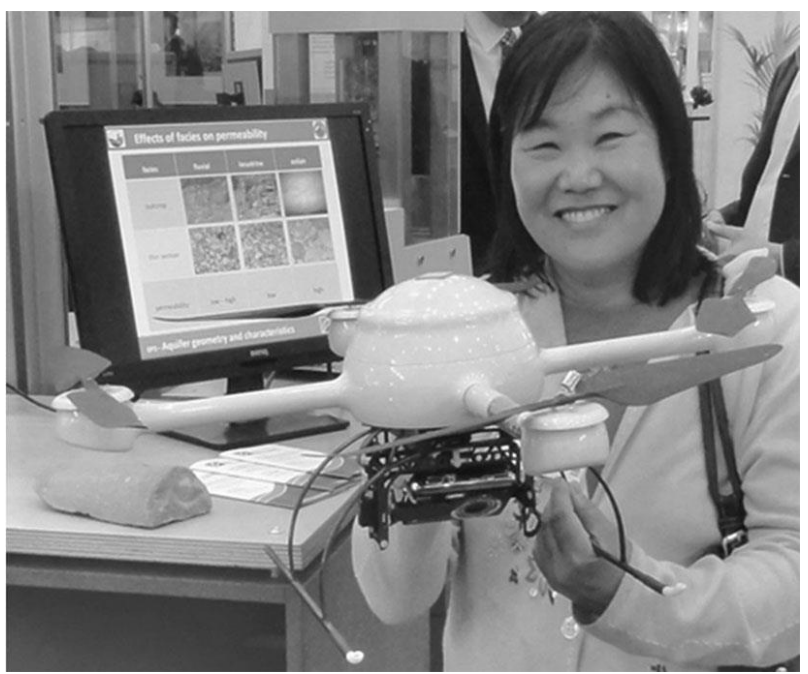

Figure 8. Currently 3D photographs in Cultural Heritage based on UAVs (civil drones) seem to promise a great future.

Though drones might carry on board GPS and allow a programming of the course, including emergency reactions, it is liked to advice, due to regulations, to extremely high risks and pointing to serious accidents, drones should be operated by skilled personal only.

3D photography in general seem to be very suited, to complete and/or at least partly to replace manual (archaeological) sketches, see also figure 1 .

In this concern the still underestimated 3D effect, which even allows, e.g., the spatial perception of extremely small scratches as well as of nuances in color differences, easy can be demonstrated, see also figure1.

For the continuously state of the art documentation of an archaeological excavation site, at least a (scaled) photographic 3D documentation should be an obligate international practice.

The (geometric) photogrammetric evaluation of these kinds of image mosaics even could be postponed.

To carry out this image processing, recently low cost "fully automated" Programme systems are available, like, e.g., the PhotoScan software, which might partly replace the 3D scanning.

The results are automatic generated textural polygonal models from still images, possibly even georeferenced. It is liked to 
emphasize, the also resulted visualization of "Structure from Motion" (SfM) is a classic sample suited for a 3D perception based on the Nuoptics method (also known as the Pulfrich or Purkinje effect) which allows the (3D)view through sunglasses with one missing glass, see table 1 .

Nevertheless it should be kept in mind, the best CAD solution cannot really compete with the original.

But, looking at the geometric and radiometric quality, a good $3 \mathrm{D}$ photograph seems to be superior to a CAD result and therefore is currently even nearer to the appearance of the original.

\section{THE COMPLETE LIST OF 3D VISUALZATION PRINCIPLES}

Furthermore a revised list of the $3 \mathrm{D}$ visualization principles, even claiming completeness, has been carried out, to decide for a particular suited 3D method, paying special attention to applications in C.H., see table 1 .

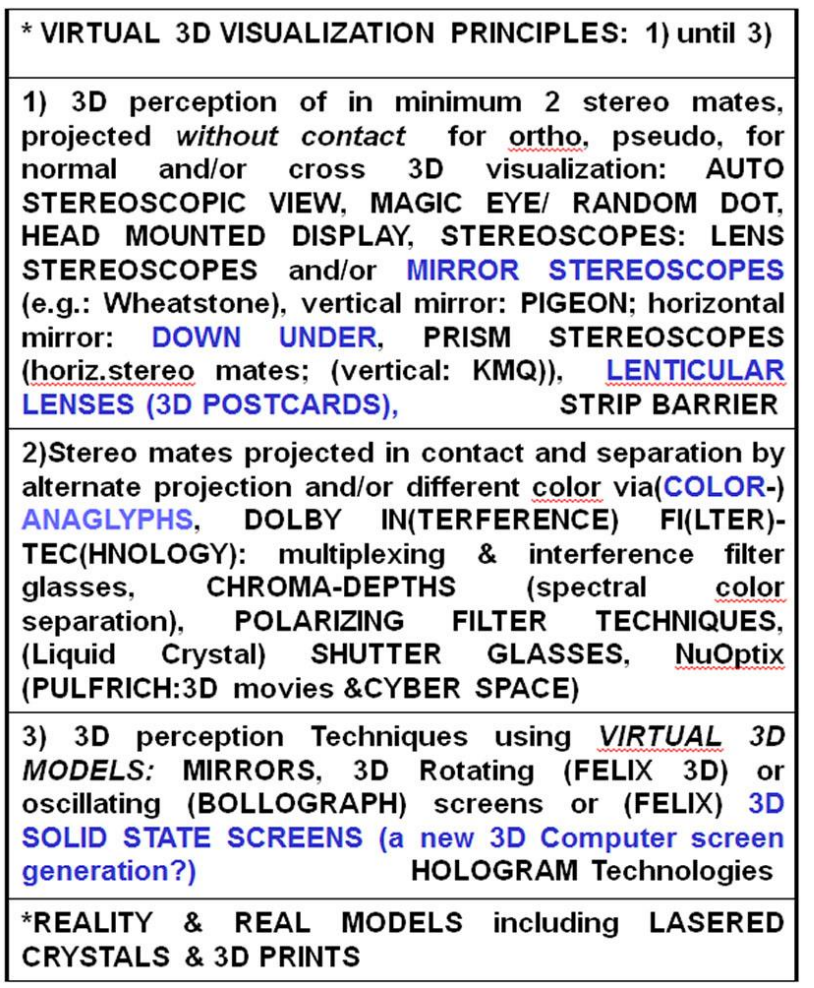

Table 1. The complete (!) list of the 3D visualization principles

\section{FUTURE OUTLOOK}

* It is highly recommended, to add the GPS coordinates and the camera orientation to every stereo pair.

In caves camera positioning and orientation can be based on gyro systems.

*Every historical and current stereo view with relevance to Cultural Heritage should appear in a global Monument Information system (MIS), like in google earth.

Beside additional information about the digital availability for this type of 3D photography, the object description, the position information, the viewing direction angle, the exposing date, the camera data and the storage media as well as the photographer should be obligate, see fig. 9 .

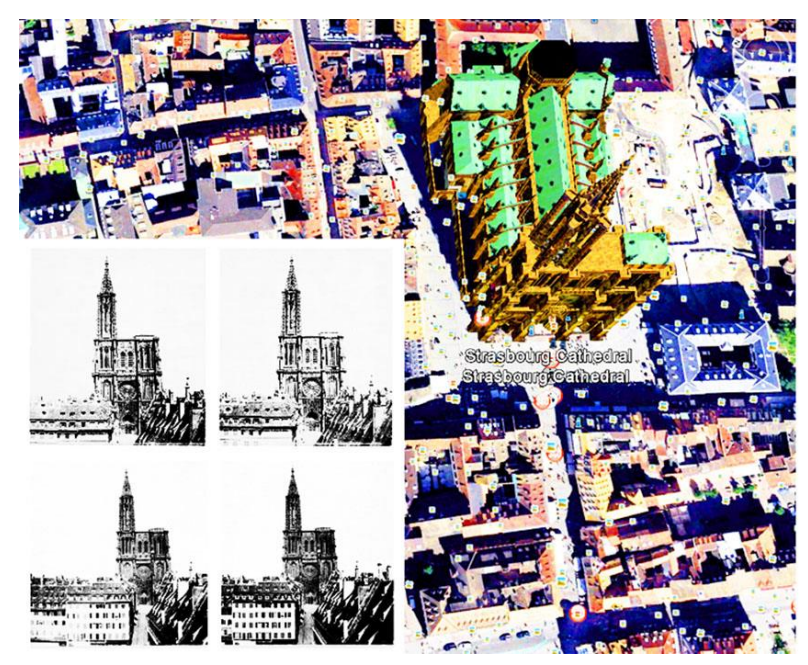

Figure 9. In addition to the access to international archives the available $3 \mathrm{D}$ photography should appear in a global GISsystem, like on, e.g., google earth.

* A consequent dealing with 3D Technology seems to indicate, currently we experience the beginning of a new age of "real 3DPC-screens", which at least could add or even partly replace the conventional 2D screens.

A first sample for this paradigm change from $2 D$ to $3 D$ screens is the FELIX solid state prototype.

Here the spatial visualization is verified without glasses in an all-around vitreous body.

In this respect nowadays widespread lasered crystals showing monuments are identified as "Early Bird"3D products, which, due to low resolution and contrast and due to lack of color, currently might even remember to the status of the invention of photography by Niepce (1827), but seem to promise a great future also in $3 \mathrm{D}$ cultural heritage documentation, compare fig. 13 to 14 .

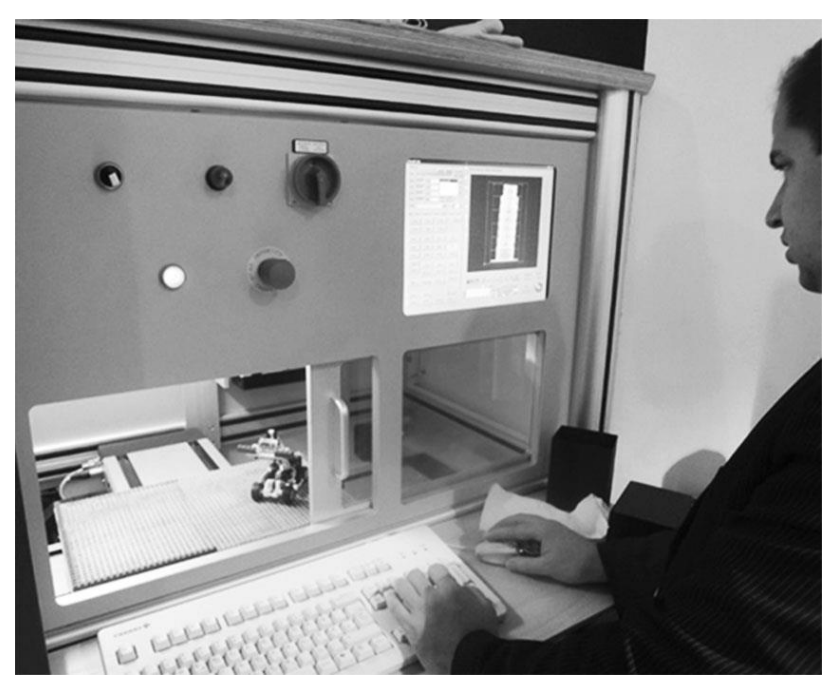

Figure 10. Sample crystal laser system (VITRO of LOOXIS (Hannover); similar: CERION) 
It is liked to point out, the transfer of the object point cloud into the crystal is verified by stimulating the crystal due to a continuously changing of the laser focus (and not by forward intersection), see fig. 10 .

A lasered crystal also allows to add "objects".

On the other hand, an object once lasered in a crystal with today's 3D technology cannot be deleted, but of course it is searched for such a (real 3D PC-screen-) material on an international level.

*Last not least currently 3D printers more and more seem to conquer the IT-market, obviously showing an international competition.

Beside others for the $3 \mathrm{D}$ printer technology the authors in particular registrated a growing spectrum (of already verified principles) with a clear tendency to a miniaturization and to decreasing in prices, see figure 11.

Beside the important 3D printing range, modern 3D printers are characterized by support variety of materials (on rolls instead of powder); auto scale mode; auto calibration; smart mode, pause and resume function as well as filament auto release system and multiple safety features; samples of 3D prints see in figure 11 to 14.

It is even anticipated, to produce "real living organic spare parts" with so called bio- or organic-printers, which currently are still in a simulation mode.

The answer to the rhetoric question, if this has any importance for Cultural Heritage might even be yes, as this could be one step for the rebirth of extinct species.

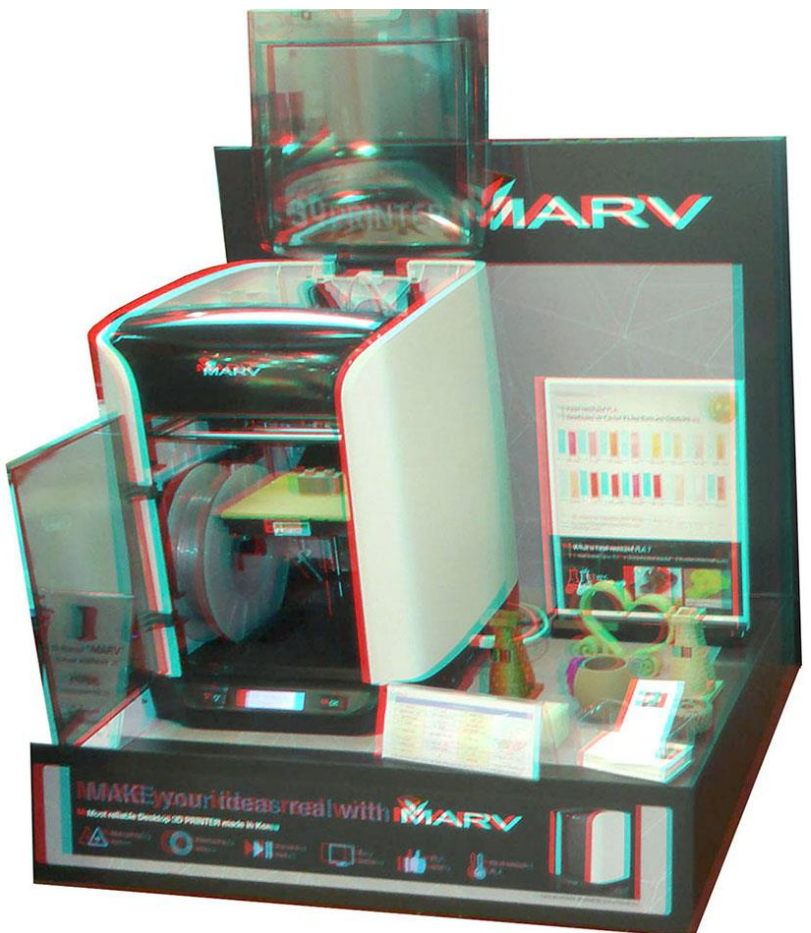

Figure 11. 3D printer (Real models also belong to the principles of $3 \mathrm{D}$ visualization)

(Courtesy MARV (South Korea), 3D photograph: Schuhr)

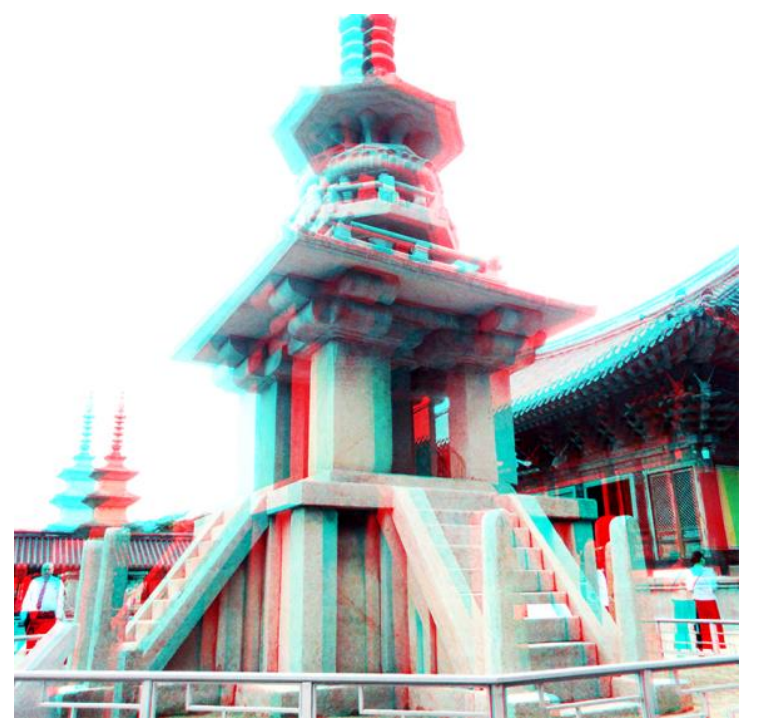

Figure 12. 3D photograph of the original of the famous Buddhistic Bulgugsa Dabotap pagoda of the former Silla Kingdom near Geongju (South Korea) (dated approx. from $\mathrm{AD} 751$ )

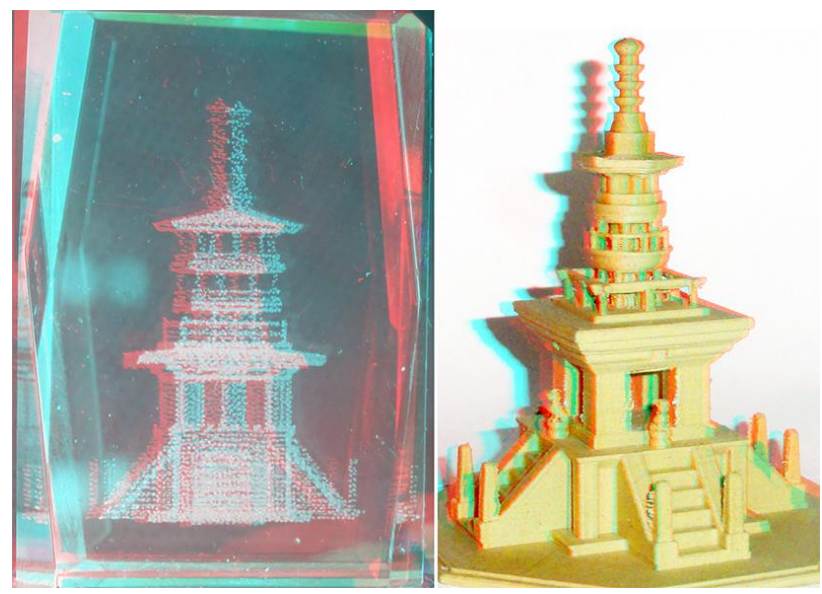

Figure 13. Comparison of a 3D photography of a laser crystal version and a $3 \mathrm{D}$ print of the Korean Dabotap pagoda as imaged in figure 12
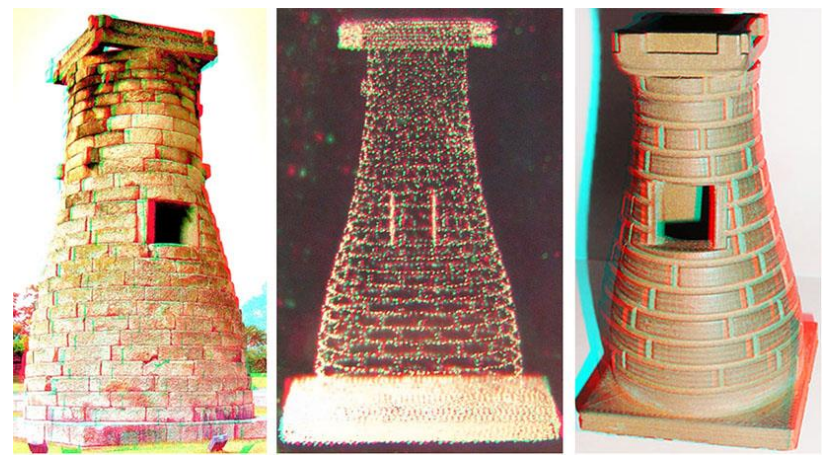

Figure 14. Comparison of a 3D photography of the original, a laser crystal version and 3D print of the world eldest observatory (Chamsongdae/South-Korea) 